\title{
On convex hulls of epigraphs of QCQPs
}

\author{
Alex L. Wang ${ }^{1}$ and Fatma Kılınç-Karzan ${ }^{1}$ \\ Carnegie Mellon University, Pittsburgh, PA, 15213, USA
}

\begin{abstract}
Quadratically constrained quadratic programs (QCQPs) are a fundamental class of optimization problems well-known to be NP-hard in general. In this paper we study sufficient conditions for a convex hull result that immediately implies that the standard semidefinite program (SDP) relaxation of a QCQP is tight. We begin by outlining a general framework for proving such sufficient conditions. Then using this framework, we show that the convex hull result holds whenever the quadratic eigenvalue multiplicity, a parameter capturing the amount of symmetry present in a given problem, is large enough. Our results also imply new sufficient conditions for the tightness (as well as convex hull exactness) of a second order cone program relaxation of simultaneously diagonalizable QCQPs.
\end{abstract}

Keywords: Quadratically constrained quadratic programming · Semidefinite program · Convex hull · Relaxation · Lagrange function .

\section{Introduction}

In this paper we study quadratically constrained quadratic programs (QCQPs) of the following form

$$
\text { Opt }:=\inf _{x \in \mathbb{R}^{N}}\left\{q_{0}(x): \begin{array}{l}
q_{i}(x) \leq 0, \forall i \in \llbracket m_{I} \rrbracket \\
q_{i}(x)=0, \forall i \in \llbracket m_{I}+1, m_{I}+m_{E} \rrbracket
\end{array}\right\},
$$

where for every $i \in \llbracket 0, m_{I}+m_{E} \rrbracket$, the function $q_{i}: \mathbb{R}^{N} \rightarrow \mathbb{R}$ is a (possibly nonconvex) quadratic function. We will write $q_{i}(x)=x^{\top} A_{i} x+2 b_{i}^{\top} x+c_{i}$ where $A_{i} \in \mathbb{S}^{N}, b_{i} \in \mathbb{R}^{N}$, and $c_{i} \in \mathbb{R}$. We will assume that the number of constraints $m:=m_{I}+m_{E}$ is at least 1 .

QCQPs arise naturally in many areas. A non-exhaustive list of applications contains facility location, production planning, pooling, max-cut, max-clique, and certain robust optimization problems (see [2, 7, 21] and references therein).

Although QCQPs are NP-hard to solve in general, they admit tractable convex relaxations. One natural relaxation is the standard (Shor) semidefinite program (SDP) relaxation [34]. There is a vast literature on approximation guarantees associated with this relaxation [9, 27, 30, 40], however, less is known about its exactness. Recently, a number of exciting results in phase retrieval [17] and clustering $[1,28,31]$ have shown that under various assumptions on the data, the QCQP formulation of the corresponding problem has a tight SDP relaxation. In contrast to these results, which address QCQPs arising from particular 
problems, Burer and Ye [16] very recently gave appealing deterministic sufficient conditions under which the standard SDP relaxation of general QCQPs is tight. In our paper, we continue this vein of research for general QCQPs. More precisely, we will provide sufficient conditions under which the convex hull of the epigraph of the QCQP is given by the projection of the epigraph of its SDP relaxation. Note that such a result immediately implies that the optimal objective value of the QCQP is equal to the optimal objective value of its SDP relaxation. We will refer to these two types of results as "convex hull results" and "SDP tightness results." In this paper we will focus mainly on conditions that imply the convex hull result. See the full paper [38] for additional new conditions which imply the SDP tightness result directly.

Convex hull results will necessarily require stronger assumptions than SDP tightness results, however they are also more broadly applicable because they may be used to derive strong convex relaxations for complex problems. In fact, the convexification of commonly occurring substructures has been critical in advancing the state-of-the-art computational approaches for mixed integer linear programs and general nonlinear nonconvex programs [18, 36]. For computational purposes, conditions guaranteeing simple convex hull descriptions are particularly favorable. As we will discuss later, a number of our sufficient conditions will guarantee that the desired convex hulls are given by a finite number of easily computable convex quadratic constraints in the original space of variables.

Related work Convex hull results are well-known for simple QCQPs such as the Trust Region Subproblem (TRS) and the Generalized Trust Region Subproblem (GTRS). Recall that the TRS is a QCQP with a single strictly convex inequality constraint and that the GTRS is a QCQP with a single (possibly nonconvex) inequality constraint. A celebrated result due to Fradkov and Yakubovich [19] implies that the SDP relaxation of the GTRS is tight. More recently, HoNguyen and Kılınç-Karzan [22] and Wang and Kılınç-Karzan [37] showed that the (closed) convex hulls of the TRS and GTRS epigraphs are given exactly by the projection of the SDP epigraphs. In both cases, the projections of the SDP epigraphs can also be described in the original space of variables with at most two convex quadratic inequalities. As a result, the TRS and the GTRS can be solved without explicitly running costly SDP-based algorithms.

A different line of research has focused on providing explicit descriptions for the convex hull of the intersection of a single nonconvex quadratic region with convex sets such as convex quadratic regions, second-order cones (SOCs), or polytopes, or with one other nonconvex quadratic region [14, 24, 29, 32, 42, 43]. For example, the convex hull of the intersection of a two-term disjunction, which is a nonconvex quadratic constraint under mild assumptions, with the second-order cone (SOC) or its cross sections has received much attention in mixed integer programming; see [14, 24, 43] and references therein. In contrast to these results, we will not limit the number of nonconvex quadratic constraints in our QCQPs. On the other hand, the nonconvex sets that we study in this paper will arise as epigraphs of QCQPs. In particular, the epigraph variable 
will play a special role in our analysis. Therefore, we view our developments as complementary to these results.

The convex hull question has also received attention for certain strengthened relaxations of simple QCQPs $[12,13,15,35]$. In this line of work, the standard SDP relaxation is strengthened by additional inequalities derived using the Reformulation-Linearization Technique (RLT). For example, Sturm and Zhang [35] showed that the standard SDP relaxation strengthened with an additional SOC constraint derived from RLT gives the convex hull of the epigraph of the TRS with one additional linear inequality. See [12] for a survey of some results in this area. In this paper, we restrict our attention to the standard SDP relaxation of QCQPs. Nevertheless, exactness conditions for strengthened SDP relaxations of QCQPs are clearly of great interest and are a direction for future research.

A number of SDP tightness results are known for variants of the TRS [6, 22, 23, 41], for simultaneously diagonalizable QCQPs [26], quadratic matrix programs [4, 5], and random general QCQPs [16]. See the full version of this paper for a more complete survey of the related SDP tightness results.

Overview and outline of paper In contrast to the literature, which has mainly focused on simple QCQPs or QCQPs under certain structural assumptions, in this paper, we will consider general QCQPs and develop sufficient conditions for both the convex hull result and the SDP tightness result.

We first introduce the epigraph of the QCQP by writing

$$
\text { Opt }=\inf _{(x, t) \in \mathbb{R}^{N+1}}\{2 t:(x, t) \in \mathcal{D}\}
$$

where $\mathcal{D}$ is the epigraph of the QCQP in (1), i.e.,

$$
\mathcal{D}:=\left\{(x, t) \in \mathbb{R}^{N} \times \mathbb{R}: \begin{array}{l}
q_{0}(x) \leq 2 t \\
q_{i}(x) \leq 0, \forall i \in \llbracket m_{I} \rrbracket \\
\\
q_{i}(x)=0, \forall i \in \llbracket m_{I}+1, m \rrbracket
\end{array}\right\} .
$$

As $(x, t) \mapsto 2 t$ is linear, we may replace the (potentially nonconvex) epigraph $\mathcal{D}$ with its convex hull $\operatorname{conv}(\mathcal{D})$. Then,

$$
\text { Opt }=\inf _{(x, t) \in \mathbb{R}^{N+1}}\{2 t:(x, t) \in \operatorname{conv}(\mathcal{D})\} .
$$

A summary of our contributions ${ }^{1}$, along with an outline of the paper, is as follows. In Section 2, we introduce and study the standard SDP relaxation of QCQPs [34] along with its optimal value $\mathrm{Opt}_{\mathrm{SDP}}$ and projected epigraph $\mathcal{D}_{\mathrm{SDP}}$. We set up a framework for deriving sufficient conditions for the "convex hull result," $\operatorname{conv}(\mathcal{D})=\mathcal{D}_{\mathrm{SDP}}$, and the "SDP tightness result," Opt $=\mathrm{Opt}_{\mathrm{SDP}}$. This framework is based on the Lagrangian function $(\gamma, x) \mapsto q_{0}(x)+\sum_{i=1}^{m} \gamma_{i} q_{i}(x)$

\footnotetext{
1 Due to space constraints, we omit full proofs, more detailed comparisons of our results with the literature, and our SDP tightness results in this extended abstract. The full version of this paper can be found at [38].
} 
and the eigenvalue structure of a dual object $\Gamma \subseteq \mathbb{R}^{m}$. This object $\Gamma$, which consists of the convex Lagrange multipliers, has been extensively studied in the literature (see [39, Chapter 13.4] and more recently [33]). In Section 3, we define an integer parameter $k$, the quadratic eigenvalue multiplicity, that captures the amount of symmetry in a given QCQP. We then give examples where the quadratic eigenvalue multiplicity is large. Specifically, vectorized reformulations of quadratic matrix programs [4] are such an example. In Section 4 , we use our framework to derive sufficient conditions for the convex hull result: $\operatorname{conv}(\mathcal{D})=\mathcal{D}_{\mathrm{SDP}}$. Theorem 2 states that if $\Gamma$ is polyhedral and $k$ is sufficiently large, then $\operatorname{conv}(\mathcal{D})=\mathcal{D}_{\text {SDP }}$. This theorem actually follows as a consequence of Theorem 1, which replaces the assumption on the quadratic eigenvalue multiplicity with a weaker assumption regarding the dimension of zero eigenspaces related to the $A_{i}$ matrices. Furthermore, our results in this section establish that if $\Gamma$ is polyhedral, then $\mathcal{D}_{\mathrm{SDP}}$ is SOC representable; see Remark 3. In particular, when the assumptions of Theorems 1 or 2 hold, we have that $\operatorname{conv}(\mathcal{D})=\mathcal{D}_{\mathrm{SDP}}$ is SOC representable. We provide several classes of problems that satisfy the assumptions of these theorems. In particular, we recover a number of results regarding the TRS [22], the GTRS [37], and the solvability of systems of quadratic equations [3].

To the best of our knowledge, our results are the first to provide a unified explanation of many of the exactness guarantees in the literature. Moreover, we provide significant generalizations of known results in a number of settings.

Notation For nonnegative integers $m \leq n$ let $\llbracket n \rrbracket:=\{1, \ldots, n\}$ and $\llbracket m, n \rrbracket:=$ $\{m, m+1, \ldots, n-1, n\}$. Let $\mathbf{S}^{n-1}=\left\{x \in \mathbb{R}^{n}:\|x\|=1\right\}$ denote the $n-1$ sphere. Let $\mathbb{S}^{n}$ denote the set of real symmetric $n \times n$ matrices. For a positive integer $n$, let $I=I_{n}$ denote the $n \times n$ identity matrix. When the dimension is clear, we will simply write $I$. Given two matrices $A$ and $B$, let $A \otimes B$ denote their Kronecker product. For a set $\mathcal{D} \subseteq \mathbb{R}^{n}$, let $\operatorname{conv}(\mathcal{D}), \operatorname{cone}(\mathcal{D}), \operatorname{extr}(\mathcal{D}), \operatorname{dim}(\mathcal{D})$ and $\operatorname{aff} \operatorname{dim}(\mathcal{D})$ denote the convex hull, conic hull, extreme points, dimension, and affine dimension of $\mathcal{D}$, respectively.

\section{A general framework}

In this section, we introduce a general framework for analyzing the standard Shor SDP relaxation of QCQPs. We will examine how both the objective value and the feasible domain change when moving from a QCQP to its SDP relaxation.

We make an assumption that can be thought of as a primal feasibility and dual strict feasibility assumption. This assumption (or a slightly stronger version of it) is standard and is routinely made in the literature on QCQPs [4, 10, 41].

Assumption 1. Assume the feasible region of (1) is nonempty and there exists $\gamma^{*} \in \mathbb{R}^{m}$ such that $\gamma_{i}^{*} \geq 0$ for all $i \in \llbracket m_{I} \rrbracket$ and $A_{0}+\sum_{i=1}^{m} \gamma_{i}^{*} A_{i} \succ 0$. 
The standard SDP relaxation to (1) is

$$
\operatorname{Opt}_{\mathrm{SDP}}:=\inf _{x \in \mathbb{R}^{N}, X \in \mathbb{S}^{N}}\left\{\begin{array}{c}
Y:=\left(\begin{array}{ll}
1 & x^{\top} \\
x & X
\end{array}\right) \\
\left\langle Q_{0}, Y\right\rangle: \\
\left\langle Q_{i}, Y\right\rangle \leq 0, \forall i \in \llbracket m_{I} \rrbracket \\
\left\langle Q_{i}, Y\right\rangle=0, \forall i \in \llbracket m_{I}+1, m \rrbracket \\
Y \succeq 0
\end{array}\right\},
$$

where $Q_{i} \in \mathbb{S}^{N+1}$ is the matrix $Q_{i}:=\left(\begin{array}{cc}c_{i} & b_{i}^{\top} \\ b_{i} & A_{i}\end{array}\right)$. Let $\mathcal{D}_{\mathrm{SDP}}$ denote the epigraph of the relaxation (3) projected away from the $X$ variables, i.e., define

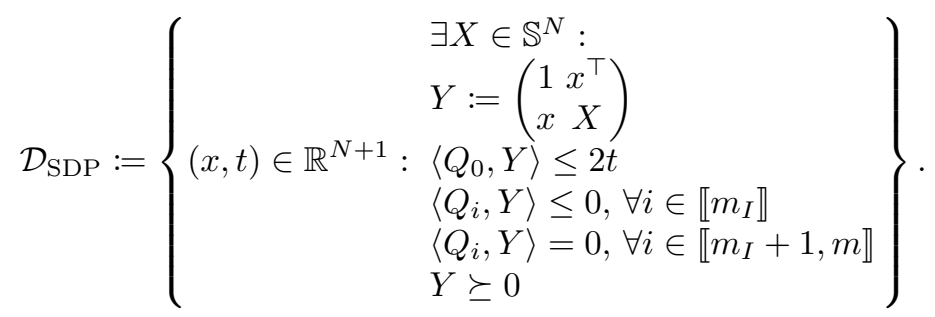

By taking $X=x x^{\top}$ in both (3) and (4), we see that $\mathcal{D} \subseteq \mathcal{D}_{\text {SDP }}$ and Opt $\geq$ Opt $_{\text {SDP }}$. Noting that $\mathcal{D}_{\mathrm{SDP}}$ is convex (it is the projection of a convex set), we further have that $\operatorname{conv}(\mathcal{D}) \subseteq \mathcal{D}_{\mathrm{SDP}}$. The framework that we set up in the remainder of this section allows us to reason about when equality occurs in either relation.

\subsection{Rewriting the SDP in terms of a dual object}

For $\gamma \in \mathbb{R}^{m}$, define

$$
\begin{aligned}
& A(\gamma):=A_{0}+\sum_{i=1}^{m} \gamma_{i} A_{i}, \quad b(\gamma):=b_{0}+\sum_{i=1}^{m} \gamma_{i} b_{i}, \quad c(\gamma):=c_{0}+\sum_{i=1}^{m} \gamma_{i} c_{i}, \\
& q(\gamma, x):=q_{0}(x)+\sum_{i=1}^{m} \gamma_{i} q_{i}(x) .
\end{aligned}
$$

Our framework for analyzing (3) is based on the dual object

$$
\Gamma:=\left\{\gamma \in \mathbb{R}^{m}: \begin{array}{l}
A(\gamma) \succeq 0 \\
\gamma_{i} \geq 0, \forall i \in \llbracket m_{I} \rrbracket
\end{array}\right\} .
$$

This object will play a key role our analysis for the following fundamental reason.

Lemma 1. Suppose Assumption 1 holds. Then

$$
\mathcal{D}_{\mathrm{SDP}}=\left\{(x, t): \sup _{\gamma \in \Gamma} q(\gamma, x) \leq 2 t\right\} \quad \text { and } \quad \operatorname{Opt}_{\mathrm{SDP}}=\min _{x \in \mathbb{R}^{N}} \sup _{\gamma \in \Gamma} q(\gamma, x) .
$$

The second identity is well-known; see e.g., Fujie and Kojima [20]. 


\subsection{The eigenvalue structure of $\Gamma$}

We now define a number of objects related to $\Gamma$. Noting that $\gamma \mapsto q(\gamma, \hat{x})$ is linear and that $\Gamma$ is closed leads to the following observation.

Observation 1 Let $\hat{x} \in \mathbb{R}^{N}$. If $\sup _{\gamma \in \Gamma} q(\gamma, \hat{x})$ is finite, then $q(\gamma, \hat{x})$ achieves its maximum value in $\Gamma$ on some face $\mathcal{F}$ of $\Gamma$.

In particular, the following definition is well-defined.

Definition 1. For any $\hat{x} \in \mathbb{R}^{N}$ such that $\sup _{\gamma \in \Gamma} q(\gamma, \hat{x})$ is finite, define $\mathcal{F}(\hat{x})$ to be the face of $\Gamma$ maximizing $q(\gamma, \hat{x})$.

Definition 2. Let $\mathcal{F}$ be a face of $\Gamma$. We say that $\mathcal{F}$ is a definite face if there exists $\gamma \in \mathcal{F}$ such that $A(\gamma) \succ 0$. Otherwise, we say that $\mathcal{F}$ is a semidefinite face and let $\mathcal{V}(\mathcal{F})$ denote the shared zero eigenspace of $\mathcal{F}$, i.e.,

$$
\mathcal{V}(\mathcal{F}):=\left\{v \in \mathbb{R}^{N}: A(\gamma) v=0, \forall \gamma \in \mathcal{F}\right\} .
$$

It is possible to show that for $\mathcal{F}$ semidefinite, the set $\mathcal{V}(\mathcal{F})$ is nontrivial. As a sketch, suppose otherwise, then for every $v$ on the unit sphere, we can associate a $\gamma_{v} \in \mathcal{F}$ such that $v^{\top} A\left(\gamma_{v}\right) v>0$. Then we can produce a positive definite matrix $A(\bar{\gamma})$ where $\bar{\gamma}$ is an "average" over the $\gamma_{v}$, a contradiction. See Lemma 2 in the full version of this paper for a formal proof.

\subsection{The framework}

Our framework consists of two parts: an "easy part" that only requires Assumption 1 to hold and a "hard part" that may require much stronger assumptions. The "easy part" consists of the following lemma and observation.

Lemma 2. Suppose Assumption 1 holds and let $(\hat{x}, \hat{t}) \in \mathcal{D}_{\mathrm{SDP}}$. If $\mathcal{F}(\hat{x})$ is a definite face of $\Gamma$, then $(\hat{x}, \hat{t}) \in \mathcal{D}$.

Observation 2 Suppose Assumption 1 holds and let $\mathcal{F}$ be a face of $\Gamma$. If $\operatorname{aff} \operatorname{dim}(\mathcal{F})=$ $m$, then $\mathcal{F}$ is definite.

The "hard part" of the framework works as follows: In order to show the convex hull result $\mathcal{D}_{\mathrm{SDP}}=\operatorname{conv}(\mathcal{D})$, it suffices to guarantee that every $(\hat{x}, \hat{t}) \in$ $\mathcal{D}_{\text {SDP }}$ can be decomposed as a convex combination of pairs $\left(x_{\alpha}, t_{\alpha}\right)$ for which $\mathcal{F}\left(x_{\alpha}\right)$ is definite. Then, by Lemma 2 , we will have that $\left(x_{\alpha}, t_{\alpha}\right) \in \mathcal{D}$. We give examples of such sufficient conditions in Section 4. Our decomposition procedures will be recursive and we will use Observation 2 to show that they terminate.

Remark 1. Consider performing an invertible affine transformation on the space $\mathbb{R}^{N}$, i.e. let $y=U(x+z)$ where $U \in \mathbb{R}^{N \times N}$ is an invertible linear transformation and $z \in \mathbb{R}^{N}$. Define the quadratic functions $q_{0}^{\prime}, \ldots, q_{m}^{\prime}: \mathbb{R}^{N} \rightarrow \mathbb{R}$ such that $q_{i}^{\prime}(y)=q_{i}^{\prime}(U(x+z))=q_{i}(x)$ for all $x \in \mathbb{R}^{N}$. We will use an apostrophe to denote all the quantities corresponding to the QCQP in the variable $y$. 
Define the map $\ell: \mathbb{R}^{N+1} \rightarrow \mathbb{R}^{N+1}$ by $(x, t) \mapsto(U(x+z), t)$. Note that Opt $^{\prime}=$ Opt and $\operatorname{conv}\left(\mathcal{D}^{\prime}\right)=\ell(\operatorname{conv}(\mathcal{D}))$. Furthermore a straightforward application of Lemma 1 gives $\mathrm{Opt}_{\mathrm{SDP}}^{\prime}=\mathrm{Opt}_{\mathrm{SDP}}$ and $\mathcal{D}_{\mathrm{SDP}}^{\prime}=\ell\left(\mathcal{D}_{\mathrm{SDP}}\right)$. We deduce that the questions $\operatorname{conv}(\mathcal{D}) \stackrel{?}{=} \mathcal{D}_{\mathrm{SDP}}$ and Opt $\stackrel{?}{=} \mathrm{Opt}_{\mathrm{SDP}}$ are invariant under invertible affine transformation of the $x$-space. In particular, the sufficient conditions that we will present in Theorems 1 and 2 only need to hold after some invertible affine transformation. In this sense, the SDP relaxation will "find" structure in a given QCQP even if it is "hidden" by an affine transformation.

\section{Symmetries in QCQPs}

In this section, we examine a parameter $k$ that captures the amount of symmetry present in a QCQP of the form (1).

Definition 3. The quadratic eigenvalue multiplicity of a QCQP of the form (1) is the largest integer $k$ such that for every $i \in \llbracket 0, m \rrbracket$ there exists $\mathcal{A}_{i} \in \mathbb{S}^{n}$ for which $A_{i}=I_{k} \otimes \mathcal{A}_{i}$. Let $\mathcal{A}(\gamma):=\mathcal{A}_{0}+\sum_{i=1}^{m} \gamma_{i} \mathcal{A}_{i}$.

This value is well-defined: $k$ is always at least 1 as we can write $A_{i}=I_{1} \otimes \mathcal{A}_{i}$. On the other hand, $k$ must also be a divisor of $N$.

The next lemma states the crucial structure inherent in QCQPs with large quadratic eigenvalue multiplicities.

Lemma 3. If $\mathcal{F}$ is a semidefinite face of $\Gamma$, then $\operatorname{dim}(\mathcal{V}(\mathcal{F})) \geq k$.

Remark 2. In quadratic matrix programming $[4,5]$, we are asked to optimize

$$
\inf _{X \in \mathbb{R}^{n \times k}}\left\{\begin{array}{c}
\operatorname{tr}\left(X^{\top} \mathcal{A}_{0} X\right)+2 \operatorname{tr}\left(B_{0}^{\top} X\right)+c_{0}: \\
\operatorname{tr}\left(X^{\top} \mathcal{A}_{i} X\right)+2 \operatorname{tr}\left(B_{i}^{\top} X\right)+c_{i} \leq 0, \forall i \in \llbracket m_{I} \rrbracket \\
\operatorname{tr}\left(X^{\top} \mathcal{A}_{i} X\right)+2 \operatorname{tr}\left(B_{i}^{\top} X\right)+c_{i}=0, \forall i \in \llbracket m_{I}+1, m \rrbracket
\end{array}\right\},
$$

where $\mathcal{A}_{i} \in \mathbb{S}^{n}, B_{i} \in \mathbb{R}^{n \times k}$ and $c_{i} \in \mathbb{R}$ for all $i \in \llbracket 0, m \rrbracket$. We can transform this program to an equivalent QCQP in the vector variable $x \in \mathbb{R}^{n k}$. Then $\operatorname{tr}\left(X^{\top} \mathcal{A}_{i} X\right)+2 \operatorname{tr}\left(B_{i}^{\top} X\right)+c_{i}=x^{\top}\left(I_{k} \otimes \mathcal{A}_{i}\right) x+2 b_{i}^{\top} x+c_{i}$, where $b_{i} \in \mathbb{R}^{n k}$ has entries $\left(b_{i}\right)_{(t-1) n+s}=\left(B_{i}\right)_{s, t}$. In particular, the vectorized reformulation of (5) has quadratic eigenvalue value multiplicity $k$.

\section{Convex hull results}

We now present new sufficient conditions for the convex hull result $\mathcal{D}_{\mathrm{SDP}}=$ $\operatorname{conv}(\mathcal{D})$. We analyze the case where the geometry of $\Gamma$ is particularly nice.

Assumption 2. Assume that $\Gamma$ is polyhedral. 
We remark that although Assumption 2 is rather restrictive, it is general enough to cover the case where the set of quadratic forms $\left\{A_{i}\right\}_{i \in \llbracket 0, m \rrbracket}$ is diagonal or simultaneously diagonalizable - a class of QCQPs which have been studied extensively in the literature $[8,25,26]$. See the full version of this paper for convex hull and SDP tightness results without Assumption 2 as well as a discussion on the difficulties in removing it.

Our main result in this paper is the following theorem.

Theorem 1. Suppose Assumptions 1 and 2 hold. If for every semidefinite face $\mathcal{F}$ of $\Gamma$ we have $\operatorname{dim}(\mathcal{V}(\mathcal{F})) \geq \operatorname{aff} \operatorname{dim}(\{b(\gamma): \gamma \in \mathcal{F}\})+1$, then $\operatorname{conv}(\mathcal{D})=\mathcal{D}_{\text {SDP }}$.

Assumption 1 allows us to apply Lemma 2 to handle any $(\hat{x}, \hat{t}) \in \mathcal{D}_{\mathrm{SDP}}$ for which $\mathcal{F}(\hat{x})$ is definite. Therefore, in order to prove Theorem 1, it suffices to prove the following lemma.

Lemma 4. Suppose Assumptions 1 and 2 hold. Let $(\hat{x}, \hat{t}) \in \mathcal{D}_{\mathrm{SDP}}$ and let $\mathcal{F}=$ $\mathcal{F}(\hat{x})$. If $\mathcal{F}$ is a semidefinite face of $\Gamma$ and $\operatorname{dim}(\mathcal{V}(\mathcal{F})) \geq \operatorname{aff} \operatorname{dim}(\{b(\gamma): \gamma \in \mathcal{F}\})+$ 1 , then $(\hat{x}, \hat{t})$ can be written as a convex combination of points $\left(x_{\alpha}, t_{\alpha}\right)$ satisfying the following properties:

1. $\left(x_{\alpha}, t_{\alpha}\right) \in \mathcal{D}_{\mathrm{SDP}}$, and

2. $\operatorname{aff} \operatorname{dim}\left(\mathcal{F}\left(x_{\alpha}\right)\right)>$ aff $\operatorname{dim}(\mathcal{F}(\hat{x}))$.

We give a proof sketch of Lemma 4 in Appendix A.

The proof of Theorem 1 follows at once from Lemmas 2 and 4 and Observation 2. Indeed, Lemma 4 guarantees that aff $\operatorname{dim}\left(\mathcal{F}\left(x_{\alpha}\right)\right)>\operatorname{aff} \operatorname{dim}(\mathcal{F}(\hat{x}))$. Thus, by Observation 2, we will have successfully decomposed $(\hat{x}, \hat{t})$ as a convex combination of $\left(x_{\alpha}, t_{\alpha}\right)$, where $\left(x_{\alpha}, t_{\alpha}\right) \in \mathcal{D}_{\mathrm{SDP}}$ and $\mathcal{F}\left(x_{\alpha}\right)$ is definite, after at most $m-1$ rounds of applying Lemma 4 . Finally, Lemma 2 guarantees that each pair $\left(x_{\alpha}, t_{\alpha}\right)$ is an element of $\mathcal{D}$, the epigraph of the QCQP.

The next theorem follows as a corollary to Theorem 1.

Theorem 2. Suppose Assumptions 1 and 2 hold. If for every semidefinite face $\mathcal{F}$ of $\Gamma$ we have $k \geq \operatorname{aff} \operatorname{dim}(\{b(\gamma): \gamma \in \mathcal{F}\})+1$, then $\operatorname{conv}(\mathcal{D})=\mathcal{D}_{\mathrm{SDP}}$.

Remark 3. We remark that when $\Gamma$ is polyhedral (Assumption 2), the set $\mathcal{D}_{\mathrm{SDP}}$ is actually SOC representable: By the Minkowski-Weyl Theorem, we can decompose $\Gamma=\Gamma_{e}+\operatorname{cone}\left(\Gamma_{r}\right)$ where both $\Gamma_{e}$ and $\Gamma_{r}$ are polytopes. Let $\breve{q}(\gamma, x)=$ $\sum_{i=1}^{m} \gamma_{i} q_{i}(x)$. Then, by Lemma 1 we can write

$$
\mathcal{D}_{\mathrm{SDP}}=\left\{(x, t): \sup _{\gamma \in \Gamma} q(\gamma, x) \leq 2 t\right\}=\left\{(x, t): \begin{array}{l}
q\left(\gamma_{e}, x\right) \leq 2 t, \forall \gamma_{e} \in \operatorname{extr}\left(\Gamma_{e}\right) \\
\breve{q}\left(\gamma_{f}, x\right) \leq 0, \forall \gamma_{f} \in \operatorname{extr}\left(\Gamma_{r}\right)
\end{array}\right\} .
$$

That is, $\mathcal{D}_{\mathrm{SDP}}$ is defined by finitely many convex quadratic inequalities. Thus the assumptions of Theorems 1 and 2 imply that $\operatorname{conv}(\mathcal{D})$ is SOC representable.

We now give examples of problems where our assumptions hold.

Corollary 1. Suppose $m=1$ and Assumption 1 holds. Then, $\operatorname{conv}(\mathcal{D})=\mathcal{D}_{\mathrm{SDP}}$. 
Corollary 1 recovers results associated with the epigraph of the TRS ${ }^{2}$ and the GTRS (see [22, Theorem 13] and [37, Theorems 1 and 2]).

Corollary 2. Suppose Assumptions 1 and 2 hold. If $b_{i}=0$ for all $i \in \llbracket m \rrbracket$, then $\operatorname{conv}(\mathcal{D})=\mathcal{D}_{\mathrm{SDP}}$.

Example 1. Consider the following optimization problem.

$$
\inf _{x \in \mathbb{R}^{2}}\left\{\begin{array}{ll}
x_{1}^{2}+x_{2}^{2}+10 x_{1}: & \begin{array}{l}
x_{1}^{2}-x_{2}^{2}-5 \leq 0 \\
-x_{1}^{2}+x_{2}^{2}-50 \leq 0
\end{array}
\end{array}\right\}
$$

We check that the conditions of Corollary 2 hold. Assumption 1 holds as $A(0)=$ $A_{0}=I \succ 0$ and $x=0$ is feasible. Next, Assumption 2 holds as

$$
\Gamma=\left\{\gamma \in \mathbb{R}^{2}: \begin{array}{l}
1+\gamma_{1}-\gamma_{2} \geq 0 \\
1-\gamma_{1}+\gamma_{2} \geq 0 \\
\gamma \geq 0
\end{array}\right\} .
$$

One can verify that $\Gamma=\operatorname{conv}(\{(0,0),(1,0),(0,1)\})+\operatorname{cone}(\{(1,1)\})$. Finally, we note that $b_{1}=b_{2}=0$. Hence, Corollary 2 and Remark 3 imply that

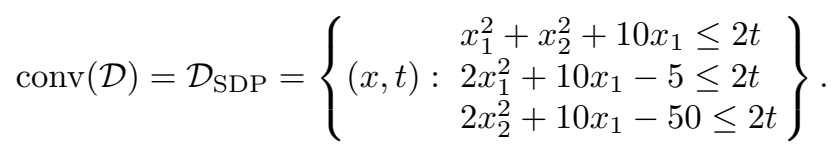

We plot $\mathcal{D}$ and $\operatorname{conv}(\mathcal{D})=\mathcal{D}_{\text {SDP }}$ in Figure 1 .
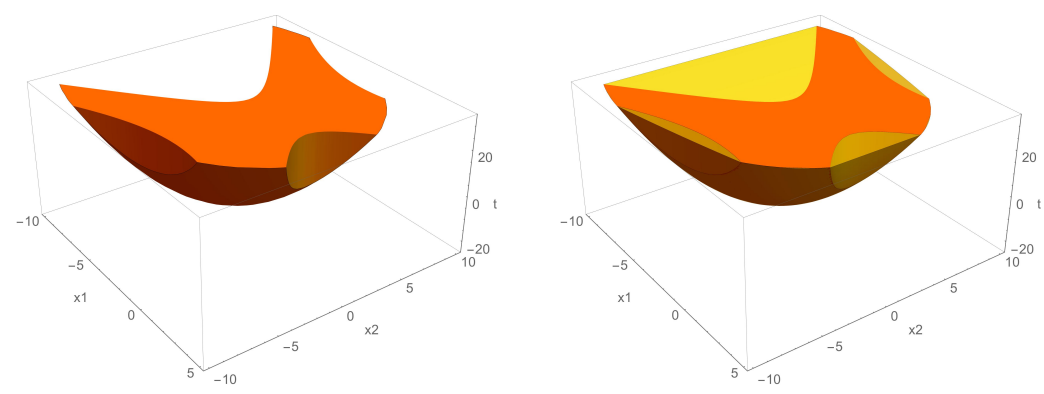

Fig. 1. The sets $\mathcal{D}$ (in orange) and $\operatorname{conv}(\mathcal{D})$ (in yellow) from Example 1

Remark 4. Barvinok [3] shows that one can decide in polynomial time (in $N$ ) whether a constant number, $m_{E}$, of quadratic forms $\left\{A_{i}\right\}_{i \in \llbracket m_{E} \rrbracket}$ has a joint nontrivial zero. That is, whether the system $x^{\top} A_{i} x=0$ for $i \in \llbracket m_{E} \rrbracket$ and $x^{\top} x=$

2 Corollary 1 fails to recover the full extent of [22, Theorem 13]. Indeed, [22, Theorem 13] also gives a description of the convex hull of the epigraph of the TRS with an additional conic constraint under some assumptions. 
1 is feasible. We can recast this as asking whether the following optimization problem

$$
\min _{x \in \mathbb{R}^{N}}\left\{-x^{\top} x: \begin{array}{l}
x^{\top} x \leq 1 \\
x^{\top} A_{i} x=0, \forall i \in \llbracket m_{E} \rrbracket
\end{array}\right\}
$$

has objective value -1 or 0 .

Thus, the feasibility problem studied in [3] reduces to a QCQP of the form we study in this paper. It is easy to verify that Assumption 1 holds. Then when $\Gamma$ is polyhedral (Assumption 2), Corollary 2 implies that the feasibility problem (even in a variable number of quadratic forms) can be decided using a semidefinite programming approach. Nevertheless, Assumption 2 may not necessarily hold in general and so Corollary 2 does not recover the full result of [3].

Corollary 3. Suppose Assumption 1 holds and for every $i \in \llbracket 0, m \rrbracket$, there exists $\alpha_{i}$ such that $A_{i}=\alpha_{i} I_{N}$. If $m \leq N$, then $\operatorname{conv}(\mathcal{D})=\mathcal{D}_{\mathrm{SDP}}$.

Remark 5. Consider the problem of finding the distance between the origin $0 \in$ $\mathbb{R}^{N}$ and a piece of Swiss cheese $C \subseteq \mathbb{R}^{N}$. We will assume that $C$ is nonempty and defined as

$$
C=\left\{\begin{array}{ll}
x \in \mathbb{R}^{N}: & \left\|x-y_{i}\right\| \leq s_{i}, \forall i \in \llbracket m_{1} \rrbracket \\
& \left.\left\|x-z_{i}\right\| \geq t_{i}, \forall i \in \llbracket m_{2}\right\rangle \geq c_{i}, \forall i \in \llbracket m_{3} \rrbracket
\end{array}\right\},
$$

where $y_{i}, z_{i}, b_{i} \in \mathbb{R}^{N}$ and $s_{i}, t_{i}, c_{i} \in \mathbb{R}$ are arbitrary. In other words, $C$ is defined by $m_{1}$-many "inside-ball" constraints, $m_{2}$-many "outside-ball" constraints, and $m_{3}$-many linear inequalities. Note that each of these constraints may be written as a quadratic inequality constraint with quadratic form $I,-I$, or 0 . In particular, Corollary 3 implies that if $m_{1}+m_{2}+m_{3} \leq N$, then the value

$$
\inf _{x \in \mathbb{R}^{N}}\left\{\|x\|^{2}: x \in C\right\}
$$

may be computed using the standard SDP relaxation of the problem.

Bienstock and Michalka [11] give sufficient conditions under which a related problem

$$
\inf _{x \in \mathbb{R}^{N}}\left\{q_{0}(x): x \in C\right\},
$$

is polynomial-time solvable. Here, $q_{0}: \mathbb{R}^{N} \rightarrow \mathbb{R}$ may be an arbitrary quadratic function however $m_{1}$ and $m_{2}$ must be constant. They devise an enumerative algorithm for this problem and prove its correctness under different assumptions. In contrast, our work deals only with the standard SDP relaxation and does not assume that the number of quadratic forms is constant.

\section{Acknowledgments}

This research is supported in part by NSF grant CMMI 1454548 . 


\section{A Proof sketch of Lemma 4}

For simplicity, we will assume that $\Gamma$ is a polytope in this proof sketch. Let $(\hat{x}, \hat{t})$ satisfy the assumptions of Lemma 4 . Without loss of generality, we may assume that $\sup _{\gamma \in \Gamma} q(\gamma, \hat{x})=2 \hat{t}$.

We claim that the following system in variables $v$ and $s$

$$
\left\{\begin{array}{l}
\langle b(\gamma), v\rangle=s, \forall \gamma \in \mathcal{F} \\
v \in \mathcal{V}(\mathcal{F}), s \in \mathbb{R}
\end{array}\right.
$$

has a nonzero solution. Indeed, we may replace the first constraint with at most

$$
\operatorname{aff} \operatorname{dim}(\{b(\gamma): \gamma \in \mathcal{F}\})+1 \leq \operatorname{dim}(\mathcal{V}(\mathcal{F}))
$$

homogeneous linear equalities in the variables $v$ and $s$. The claim then follows by noting that the equivalent system is an under-constrained homogeneous system of linear equalities and thus has a nonzero solution $(v, s)$. It is easy to verify that $v \neq 0$ and hence, by scaling, we may take $v \in \mathbf{S}^{N-1}$.

We will modify $(\hat{x}, \hat{t})$ in the $(v, s)$ direction. For $\alpha \in \mathbb{R}$, define

$$
\left(x_{\alpha}, t_{\alpha}\right):=(\hat{x}+\alpha v, \hat{t}+\alpha s) .
$$

We will sketch the existence of an $\alpha>0$ such that $\left(x_{\alpha}, t_{\alpha}\right)$ satisfies the conclusions of Lemma 4. A similar line of reasoning will produce an analogous $\alpha<0$. This will complete the proof sketch.

Suppose $\gamma \in \mathcal{F}$. Then, by our choice of $v$ and $s$, the function $\alpha \mapsto q\left(\gamma, x_{\alpha}\right)-$ $2 t_{\alpha}=q(\gamma, \hat{x})-2 t=0$ is identically zero. Now suppose $\gamma \in \Gamma \backslash \mathcal{F}$. Then, the function $\alpha \mapsto q\left(\gamma, x_{\alpha}\right)-2 t_{\alpha}$ is a convex quadratic function which is negative at $\alpha=0$.

We conclude that the following set

$$
\mathcal{Q}:=\left\{\alpha \mapsto q\left(\gamma, x_{\alpha}\right)-2 t_{\alpha}: \gamma \in \operatorname{extr}(\Gamma)\right\} \backslash\{0\},
$$

consists of convex quadratic functions which are negative at $\alpha=0$. The finiteness of this set follows from the assumption that $\Gamma$ is polyhedral.

Assumption 1 implies that at least one of the functions in $\mathcal{Q}$ is strictly convex. Then as $\mathcal{Q}$ is a finite set, there exists an $\alpha_{+}>0$ such that $q\left(\alpha_{+}\right) \leq 0$ for all $q \in \mathcal{Q}$ with at least one equality. We emphasize that this is the step where Assumption 2 cannot be dropped.

Finally, it is easy to check that $\left(x_{\alpha_{+}}, t_{\alpha_{+}}\right)$satisfies the conclusions of Lemma 4. 


\section{Bibliography}

[1] Abbe, E., Bandeira, A.S., Hall, G.: Exact recovery in the stochastic block model. IEEE Transactions on Information Theory 62(1), 471-487 (2015)

[2] Bao, X., Sahinidis, N.V., Tawarmalani, M.: Semidefinite relaxations for quadratically constrained quadratic programming: A review and comparisons. Mathematical programming 129(1), 129 (2011)

[3] Barvinok, A.I.: Feasibility testing for systems of real quadratic equations. Discrete \& Computational Geometry 10(1), 1-13 (1993)

[4] Beck, A.: Quadratic matrix programming. SIAM Journal on Optimization 17(4), 1224-1238 (2007)

[5] Beck, A., Drori, Y., Teboulle, M.: A new semidefinite programming relaxation scheme for a class of quadratic matrix problems. Operations Research Letters 40(4), 298-302 (2012)

[6] Beck, A., Eldar, Y.C.: Strong duality in nonconvex quadratic optimization with two quadratic constraints. SIAM Journal on Optimization 17(3), 844$860(2006)$

[7] Ben-Tal, A., El Ghaoui, L., Nemirovski, A.: Robust Optimization. Princeton University Press, Princeton Series in Applied Mathematics, Philadelphia, PA, USA (2009)

[8] Ben-Tal, A., den Hertog, D.: Hidden conic quadratic representation of some nonconvex quadratic optimization problems. Mathematical Programming 143(1), 1-29 (2014)

[9] Ben-Tal, A., Nemirovski, A.: Lectures on Modern Convex Optimization. MPS-SIAM Series on Optimization, SIAM, Philadehia, PA, USA (2001)

[10] Ben-Tal, A., Teboulle, M.: Hidden convexity in some nonconvex quadratically constrained quadratic programming. Mathematical Programming 72(1), 5163 (1996)

[11] Bienstock, D., Michalka, A.: Polynomial solvability of variants of the trustregion subproblem. In: Proceedings of the Twenty-Fifth Annual ACM-SIAM Symposium on Discrete Algorithms, pp. 380-390 (2014)

[12] Burer, S.: A gentle, geometric introduction to copositive optimization. Mathematical Programming 151(1), 89-116 (2015)

[13] Burer, S., Anstreicher, K.M.: Second-order-cone constraints for extended trust-region subproblems. SIAM Journal on Optimization 23(1), 432-451 (2013)

[14] Burer, S., Kılınç-Karzan, F.: How to convexify the intersection of a second order cone and a nonconvex quadratic. Mathematical Programming 162(1), 393-429 (2017)

[15] Burer, S., Yang, B.: The Trust Region Subproblem with non-intersecting linear constraints. Mathematical Programming 149(1), 253-264 (2015)

[16] Burer, S., Ye, Y.: Exact semidefinite formulations for a class of (random and non-random) nonconvex quadratic programs. Mathematical Programming pp. $1-17$ (2018) 
[17] Candes, E.J., Eldar, Y.C., Strohmer, T., Voroninski, V.: Phase retrieval via matrix completion. SIAM review 57(2), 225-251 (2015)

[18] Conforti, M., Cornuéjols, G., Zambelli, G.: Integer programming, vol. 271. Springer (2014)

[19] Fradkov, A.L., Yakubovich, V.A.: The S-procedure and duality relations in nonconvex problems of quadratic programming. Vestn. LGU, Ser. Mat., Mekh., Astron 6(1), 101-109 (1979)

[20] Fujie, T., Kojima, M.: Semidefinite programming relaxation for nonconvex quadratic programs. Journal of Global Optimization 10(4), 367-380 (Jun 1997), ISSN 1573-2916, https://doi.org/10.1023/A:1008282830093, URL https://doi.org/10.1023/A:1008282830093

[21] Phan-huy Hao, E.: Quadratically constrained quadratic programming: Some applications and a method for solution. Zeitschrift für Operations Research 26(1), 105-119 (1982)

[22] Ho-Nguyen, N., Kılınç-Karzan, F.: A second-order cone based approach for solving the Trust Region Subproblem and its variants. SIAM Journal on Optimization 27(3), 1485-1512 (2017)

[23] Jeyakumar, V., Li, G.Y.: Trust-region problems with linear inequality constraints: Exact SDP relaxation, global optimality and robust optimization. Mathematical Programming 147(1), 171-206 (2014)

[24] Kılınç-Karzan, F., Yıldız, S.: Two-term disjunctions on the second-order cone. Mathematical Programming 154(1), 463-491 (2015)

[25] Locatelli, M.: Some results for quadratic problems with one or two quadratic constraints. Operations Research Letters 43(2), 126-131 (2015)

[26] Locatelli, M.: Exactness conditions for an sdp relaxation of the extended trust region problem. Optimization Letters 10(6), 1141-1151 (2016)

[27] Megretski, A.: Relaxations of quadratic programs in operator theory and system analysis. In: Borichev, A.A., Nikolski, N.K. (eds.) Systems, Approximation, Singular Integral Operators, and Related Topics, pp. 365-392, Birkhäuser Basel, Basel (2001), ISBN 978-3-0348-8362-7

[28] Mixon, D.G., Villar, S., Ward, R.: Clustering subgaussian mixtures by semidefinite programming. arXiv preprint arXiv:1602.06612 (2016)

[29] Modaresi, S., Vielma, J.P.: Convex hull of two quadratic or a conic quadratic and a quadratic inequality. Mathematical Programming 164(1-2), 383-409 (2017)

[30] Nesterov, Y.: Quality of semidefinite relaxation for nonconvex quadratic optimization. Tech. rep., Université catholique de Louvain, Center for Operations Research and (1997)

[31] Rujeerapaiboon, N., Schindler, K., Kuhn, D., Wiesemann, W.: Size matters: Cardinality-constrained clustering and outlier detection via conic optimization. SIAM Journal on Optimization 29(2), 1211-1239 (2019)

[32] Santana, A., Dey, S.S.: The convex hull of a quadratic constraint over a polytope. arXiv preprint arXiv:1812.10160 (2018)

[33] Sheriff, J.L.: The convexity of quadratic maps and the controllability of coupled systems. Ph.D. thesis (2013) 
[34] Shor, N.Z.: Dual quadratic estimates in polynomial and boolean programming. Annals of Operations Research 25(1), 163-168 (1990)

[35] Sturm, J.F., Zhang, S.: On cones of nonnegative quadratic functions. Mathematics of Operations Research 28(2), 246-267 (2003)

[36] Tawarmalani, M., Sahinidis, N.V., Sahinidis, N.: Convexification and global optimization in continuous and mixed-integer nonlinear programming: theory, algorithms, software, and applications, vol. 65. Springer Science \& Business Media (2002)

[37] Wang, A.L., Kılınç-Karzan, F.: The generalized trust region subproblem: solution complexity and convex hull results. Tech. Rep. arXiv:1907.08843, ArXiV (2019), URL https://arxiv.org/abs/1907.08843

[38] Wang, A.L., Kılınç-Karzan, F.: On the tightness of SDP relaxations of QCQPs. Tech. Rep. arXiv:1911.09195, ArXiV (2019), URL https://arxiv.org/ abs/1911.09195

[39] Wolkowicz, H., Saigal, R., Vandenberghe, L.: Handbook of semidefinite programming: theory, algorithms, and applications, vol. 27. Springer Science \& Business Media (2012)

[40] Ye, Y.: Approximating quadratic programming with bound and quadratic constraints. Mathematical programming 84(2), 219-226 (1999)

[41] Ye, Y., Zhang, S.: New results on quadratic minimization. SIAM Journal on Optimization 14(1), 245-267 (2003)

[42] Ylldiran, U.: Convex hull of two quadratic constraints is an LMI set. IMA Journal of Mathematical Control and Information 26(4), 417-450 (2009)

[43] Yıldız, S., Cornuéjols, G.: Disjunctive cuts for cross-sections of the secondorder cone. Operations Research Letters 43(4), 432-437 (2015) 